2002), which included patients of all ages, has confirmed our findings of a higher brain weight in suicide (Salib \& Tadros, 2000) but only after they excluded 'outdoor' cases (where the body was found at an outdoor location away from the home) and controls. Hamilton \& McMahon should be congratulated on their study; however, the negative findings in the younger age group may have been confounded by the choice of the control group, some of whom may have had a mode of death not dissimilar to suicide but had a non-suicide verdict returned by the coroner. On the other hand, Hamilton \& McMahon (2002) are correct in making the assumption that our control group may have included people with pre-clinical dementia with lighter brains. This may have had the opposite effect on the findings (i.e. heavier brain weight in elderly suicide cases).

Balazic, J. \& Marušič, A. (2002) Apparent higher brain weight in suicide victims: possible reasons. Psychological Reports, 90, 236-238.

Hamilton, S. J. \& McMahon, R. F. T. (2002) Sudden death and suicide: a comparison of brain weight. British Journal of Psychiatry, I8I, 72-75.

Salib, E. \& Tadros, G. (2000) Brain weight in suicide. An exploratory study. British journal of Psychiatry, 177, 257-261.

E. Salib Liverpool University, Hollins Park Hospital, Warrington WA2 8WA, UK

\section{Vascular events associated with pharmacotherapy}

Further to an article on the prevalence of vascular events in association with the treatment of psychotic illness (Thomassen et al, 2001) and the subsequent correspondence (Curtin \& Blum, 2002), we would like to add our comments to this interesting topic. On our unit we have recently had occasion to observe a patient with haematological abnormalities that we feel were directly associated with treatment with antipsychotic medication. The case described below attests to the potential danger of therapy for schizophrenia and adds to concerns regarding the use of clozapine in particular.

Ms B., a 40-year-old woman, was receiving treatment with antipsychotic medication for recurrent episodes of agitation and psychosis. There had been a relatively poor response to trials of three antipsychotic agents and her side-effect profile was such that there were concerns about developing signs of tardive dyskinesia. A trial of clozapine was commenced and beneficial effects were apparent within 4 weeks.

Three months into treatment there was a deterioration in Ms B.'s physical condition and she was troubled by abdominal pain and continuing dyspepsia. She was evaluated and a series of blood tests were ordered. These were normal except for a very high erythrocyte sedimentation rate (ESR) of $90 \mathrm{~mm} / \mathrm{l}$, considerably above the normal for a woman of her age. The extent of the elevation was such that a battery of tests were used by our medical colleagues to establish a cause for this abnormality. Despite extensive medical investigations no abnormality was found. The ESR remained persistently elevated above $85 \mathrm{~mm} / \mathrm{l}$.

After 5 months on treatment Ms B. developed prominent visual hallucinatory experiences, which were new developments. As these resembled epileptiform discharges that were distressing for the patient, it was decided to discontinue the clozapine therapy completely. Within 2 weeks her ESR had fallen to $15 \mathrm{~mm} / \mathrm{l}$ and it has not been found to be outside the normal range since that time.

This case suggests that clozapine can produce changes in ESR, which is a crude marker of coagulation status. The persistent change seen in the ESR in this patient could not be explained by any disease process, and it certainly points to the possibility that the clozapine was implicated in increasing her blood viscosity. As a raised ESR is associated with hypercoagulability states such as those seen in malignancies, this must be a source of concern. We are pursuing our interest in this area further.

Curtin, F. \& Blum, M. (2002) Antipsychotics and risk of venous thrombosis (letter). British Journal of Psychiatry 180, 85

Thomassen, R., Vandenbroucke, J. P. \& Rosendaal, F. R. (200I) Antipsychotic medication and venous thrombosis. British Journal of Psychiatry, 179, 63-66.

P. Murphy, A. Roche, A. Byrne Department of Psychiatry, Naas General Hospital, Co. Kildare, Ireland

\section{Outcome measurement in mental health: the Italian experience in psychogeriatrics}

We would like to comment on the interesting editorial by Holloway (2002) on outcome measurement in mental health, by reporting observations on psychogeriatric services in Italy.

Our country is going through a profound economic crisis, with consequences for health expenditure. In this framework regional governments, who have the duty to coordinate health service programmes, are induced to save money in the more 'frail' areas, such as mental health and geriatric services. One of the reasons for this attitude is the claim that evidence for the usefulness of these services is weak. Yet, at the same time, there are very few attempts to fund programmes devoted to acquiring such evidence. Only a small proportion of research grants, even from central government, are devoted to outcomes research in this area. We argue that the scarcity of health resources combined with devolution of health care from central to local governments support an urgent need for outcomes research implementation in the psychogeriatric field.

At a conservative estimate, $<20 \%$ of the procedures adopted in psychogeriatrics are evidence-based and follow accepted guidelines. We agree about 'the difficulty of conducting evaluation of the complex social interventions typically deployed within mental [and, we would add, geriatric] health services' (Holloway, 2002) but, paradoxically, it is in times of scarce resources that it is of most relevance to evaluate whether the allocation of money to psychogeriatric services leads to significantly improved outcomes. Moreover, the 21 regions of Italy are undergoing a process of autonomy. One of the risks of this is that each region will adopt different means of measuring the quality of procedures and outcomes. This is particularly relevant if we consider the fact that evidence-based medicine, which might be a standard reference, covers only a small proportion of interventions.

We do not have programmes similar to the UK Department of Health's 'Mental Health Information Strategy' nor do we collect data to compile a minimum dataset. The majority of the work in psychogeriatrics is done without quality controls and it is not possible to benchmark different services against each other. Furthermore, clinicians are deprived of the possibility of measuring outcomes of their interventions, particularly in areas where the data do not allow a direct transfer of information in everyday clinical practice. 